\title{
AN EXPERIMENTAL ASSESSMENT OF LIFI DATA COMMUNICATION
}

\author{
V. Gordon And P. DANQuAH* \\ (Council for Scientific and Industrial Research-Institute for \\ Scientific and Technological Information, Accra, Ghana). \\ *Corresponding author's email: pauldanquah@yahoo.com
}

\begin{abstract}
In this paper, two functional light fidelity real-time testbeds implemented on custom Light fidelity (LiFi) kits are presented. This paper evaluates the use of LiFi technology over different distances and angular placement of illuminating devices and endpoint transceivers with connectivity locally and to the internet. The objective is to determine if distance or angular positioning in LiFi influence speeds or not. Finally, it aims to contribute to knowledge in the LiFi communication domain. We were limited to developing a customized testbed for wireless optical communication by accessing data in the physical layer via photons. In a lateral distance and angular position, we examined the performance of a laptop with LiFi activated by a $3730 \mathrm{~lm}$ LED. The setup evaluated the performance of our design in a downlink and uplink scenario where the transmitter was embedded in the main LED unit and the laptop was used as a photoreceiver. The data rate $/ \mathrm{kbps}$, the lateral distance $/ \mathrm{m}$, and the angle displacement $/ \theta^{\circ}$ were the comparison metrics. The results of the tests show that the transmission of LiFi is not influenced by distance and angular positioning for both downloads and uploads over different distances.
\end{abstract}

Keywords: Light fidelity, spectral congestion, photodetector, optical channel, testbed, data transmission.

\section{Introduction}

Wireless technologies are used globally by individuals, the private and public sectors for both voice and data communication. They have become indispensable support and a driver for information flows in the economy and provide users with access to a very large quantity of information and services. Since the popularization of the internet over wireless technologies in the recent years, the development of the optical wireless technology has greatly increased the possibilities of pervasive data access, making it possible to deliver multi-media content to consumers and incorporate the internet even further into business operations and services.
Humanity has depended on the visible light for vision and illumination due to the inherent affinity of the human eye to interpret the wavelengths associated with it. Light fidelity utilizes the vast potential of the unused, unlicensed, bio-friendly visible light portion of the electromagnetic spectrum (Haas, 2013). The wide bandwidth of this region, $380 \mathrm{~nm}$ to $780 \mathrm{~nm}$ in wavelength and 10,000 times larger than the entire radio frequency spectrum, has almost no limitations on capacity. This has led to an increased in attention by the research community and the industry because of the advantages, in particular, the usage of the freely available bandwidth. 
The current trend of wireless communication with the ever-increasing demand for higher throughput and ubiquitous coverage is setting a high demand for spectrum utilization of the radio frequency communication. It is estimated that there will be 29.3 billion networked devices by 2023, up from 18.4 billion in 2018 with the momentum of Wifi hotspots growing by 13-fold from 2020 to 2023 and will cover up to 11 percent of all public Wi-Fi hotspots (Cisco VNI mobile, 2020). This observation calls for alternative means for capacity provision and extra capability communication methods in the future. The visible light part of the radio spectrum which provides about 400 $\mathrm{THz}$ of unlicensed frequency is a possible candidate that can be tapped and used for communications purposes. The market for VLC technology is estimated to reach USD 75.00 billion by 2023 , at a CAGR of $96.57 \%$ between 2018 and 2023. Factors driving this growth are faster and safer data transfer, RF spectrum bandwidth crunch, and less energy consumption (Marketsandmarkets, 2018).

$\mathrm{LiFi}$ is an application of optical wireless technology also known as visible light communication technology. This paper focuses to explore this unfolding technology and give a relative study of LiFi with other wireless communication technologies such as Wifi. Wifi is perfect for transmission of data having wireless coverage within buildings. But LiFi provides a more secure bio-friendly communication with better efficiency, higher bandwidth, availability with a very high speed, and a significantly greater spatial reuse of bandwidth. The benefits of LiFi are well known, some of these are namely; the same LED bulb used as a source of light can be used to transmit data, thus making it efficient in terms of costs and energy. It is essentially available anywhere there is a light source and is also considered secure based on being accessible by users only within the reach of the light illumination.
(Monisha \& Sudheendra, 2018) explored the LiFi technology and give a relative study with other wireless communication technologies such as Wifi. Wifi is perfect for transmission of data having wireless coverage within buildings. It was discovered that $\mathrm{LiFi}$ provides better efficiency, higher bandwidth, better security, and availability at a very high speed. (Islam \& Mondal, 2019) proposed a hybrid orthogonal frequency division multiplexing (OFDM) modulation termed as hybrid DC-biased asymmetrically-clipped pulse-amplitude-modulated OFDM (HDAP-OFDM) for providing power-efficient communication and dimming control for light fidelity schemes. Furthermore, an algorithm was presented to simulate the dimming control of HDAPOFDM to determine the optimal provision of power-efficient communication using LiFi.

The research work on LiFi cross-connection node model using whispering gallery mode of light in a microring resonator was instructive using an optical micro/nano-electro-mechanical system (OMEMS/ONEMS) is designed and simulated. (Punthawanunt, Aziz, Phatharacorn, Ali \& Yupapin, 2018). Further to this, work on IoT devices and storing securely on the cloud using LiFi reveals a strategy that permits exchanging gathered loT information over the cloud safely utilizing LiFi innovation by applying role-based access control approaches and the cryptography techniques. (Bokefode, Ubale \& Gaikwad, 2018)

(Pikasis \& Popoola, 2018) on the other hand, discussed a framework for investigating the effects of different LiFi modulation techniques on the emitted light quality of LEDs is presented. It is a valuable tool for designing LiFi systems that are compliant with lighting and data communication requirements. Singh, Sood, Thakur, Arora \& Kumar (2017), proposed the LiFi technology to design and implement a wireless communication system for toll collection. The technology is intended to 
help preserve the fuel consumption of vehicles and will create an eco-friendly environment.

Mahendran (2017) confirmed the benefits of integrated LiFi for smart communication through illumination as enumerated earlier.

This research work uses an experimental approach, the LiFi network is set up with local connectivity to an FTP server and using a LiFi kit, LiFiNET. The tests are carried out based on angles and distance of the photo transmitter (Main LED unit) to the photoreceiver (LiFi enabled Laptop). The metrics for comparison are the data rates for upload and download data access, the lateral distance variation, and the angular displacement of the photoreceiver from the photo transmitter. Details of the setup and framework for the experiment are found in Table 1:

TABLE 1

Framework for Measurement

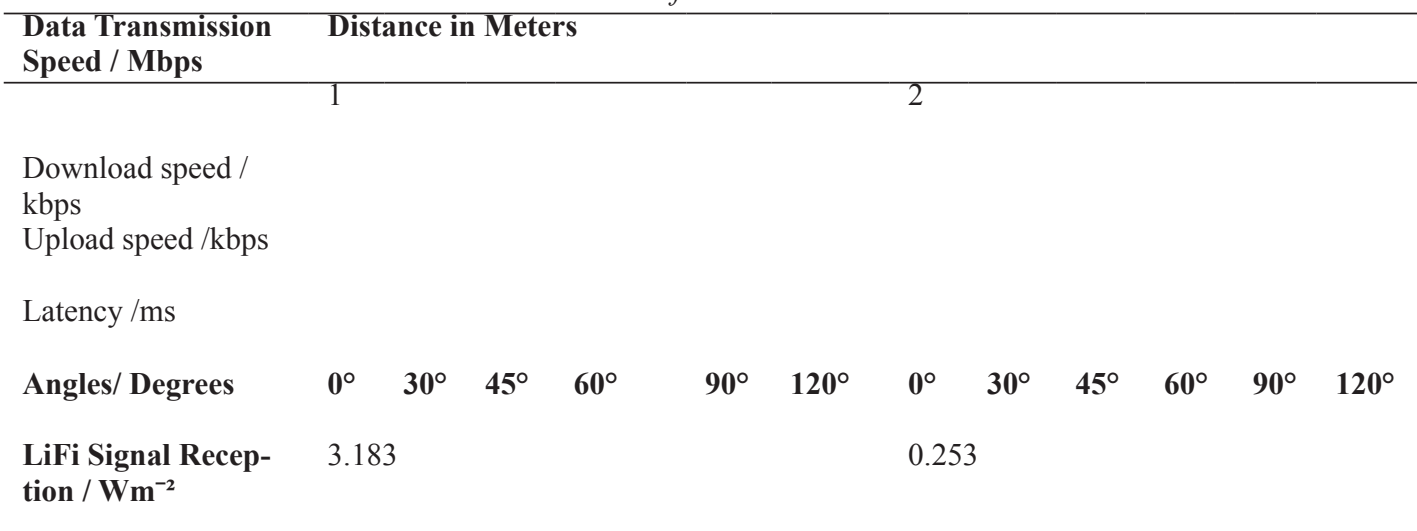

System design and simulations

The development of this project started with simulations in MATLAB of a reference LiFi modulator and demodulator according to the specifications for physical sublayer 2 (PHY 2) operating mode (IEEE, 2018). The study of the model in MATLAB serves as a benchmark for subsequent implementation. The simulation is performed on the channel only as described below.

\section{Li-Fi physical layer overview}

The physical layer (PHY) and media access control (MAC) layer are defined by the IEEE 802.15.7 standard (IEEE, 2018). This delivers enough data rates to support audio-video, and multimedia services for the short-range optical wireless communication. The Attributes of the
MAC sublayer include support for color function, color stabilization, dimming, and visibility to maintain the illumination function in the idle state and mitigate flicker (IEEE, 2018). It also handles layer 1 resource contention issues, addressing and data acknowledgment protocols. The physical layer, PHY is subdivided into PHY1, PHY2, and PHY3 sublayers. The PHY1 sublayer is used to model outdoor applications that require low data rates $(11.67 \mathrm{~kb} / \mathrm{s}$ to $266.6 \mathrm{~kb} / \mathrm{s}$ ) (IEEE, 2018), PHY2 sublayer to model indoor applications that require high data rates $(1.25 \mathrm{Mb} / \mathrm{s}$ to $96 \mathrm{Mb} / \mathrm{s})$ and PHY3 sublayer for applications where red, green and blue (RGB) LED detectors and sources are available(IEEE, 2018). For our application, we shall leverage PHY 2 which is optimized for indoor applications. 
The modulation schemes used are on-off-keying $(\mathrm{OOK})$ and variable pulse position modulation (VPPM). Reed Solomon (RS) and Convolutional encoders are proposed by the IEEE standard to correct channel errors and improve the link reliability. The physical sublayers are shown in Tables 2 and 3:

TABLE 2

PHY Sublayer 1 Operating Modes

\begin{tabular}{|c|c|c|c|c|c|}
\hline \multirow{2}{*}{$\begin{array}{l}\text { Modulation } \\
\text { OOK }\end{array}$} & \multirow{2}{*}{$\begin{array}{c}\text { RLL Code } \\
\text { Manchester }\end{array}$} & \multirow{2}{*}{$\begin{array}{c}\begin{array}{c}\text { Optical- } \\
\text { clock rate }\end{array} \\
200 \mathrm{KHZ}\end{array}$} & \multicolumn{2}{|c|}{ FEC } & \multirow[t]{2}{*}{ Data rate } \\
\hline & & & Outer code $(\mathrm{RS})$ & Inner code $(\mathrm{CC})$ & \\
\hline \multirow{9}{*}{ VPPM } & \multirow{9}{*}{$4 \mathrm{~B} 6 \mathrm{~B}$} & \multirow{9}{*}{$400 \mathrm{KHZ}$} & $\mathrm{RS}(64,32)$ & $1 / 4$ & $11.67 \mathrm{~kb} / \mathrm{s}$ \\
\hline & & & $\mathrm{RS}(160,128)$ & $1 / 3$ & $24.44 \mathrm{~kb} / \mathrm{s}$ \\
\hline & & & $\operatorname{RS}(64,32)$ & $2 / 3$ & $48.89 \mathrm{~kb} / \mathrm{s}$ \\
\hline & & & $\operatorname{RS}(160,128)$ & None & $73.3 \mathrm{~kb} / \mathrm{s}$ \\
\hline & & & $\operatorname{RS}(64,32)$ & None & $100 \mathrm{~kb} / \mathrm{s}$ \\
\hline & & & $\operatorname{RS}(160,128)$ & $1 / 4$ & $35.56 \mathrm{~kb} / \mathrm{s}$ \\
\hline & & & $\operatorname{RS}(64,32)$ & None & $71.11 \mathrm{~kb} / \mathrm{s}$ \\
\hline & & & $\operatorname{RS}(160,128)$ & None & $124.4 \mathrm{~kb} / \mathrm{s}$ \\
\hline & & & None & None & $266.6 \mathrm{~kb} / \mathrm{s}$ \\
\hline
\end{tabular}

Source: (IEEE)

TABLE 3

PHY Sublayer 2 Operating Modes

\begin{tabular}{|c|c|c|c|c|}
\hline Modulation & RLL Code & $\begin{array}{l}\text { Optical } \\
\text { clock rate }\end{array}$ & FEC & Data rate \\
\hline \multirow{6}{*}{ VPPM } & \multirow{6}{*}{ 4B6B } & $3.75 \mathrm{MHz}$ & $\mathrm{RS}(64,32)$ & $1.25 \mathrm{Mb} / \mathrm{s}$ \\
\hline & & $7.5 \mathrm{MHz}$ & $\begin{array}{l}\mathrm{RS}(160,128) \\
\operatorname{RS}(64,32)\end{array}$ & $\begin{array}{l}2 \mathrm{Mb} / \mathrm{s} \\
25 \mathrm{Mb} / \mathrm{s}\end{array}$ \\
\hline & & & $\operatorname{RS}(160,128)$ & $4 \mathrm{Mb} / \mathrm{s}$ \\
\hline & & & None & $5 \mathrm{Mb} / \mathrm{s}$ \\
\hline & & $15 \mathrm{MHz}$ & $\mathrm{RS}(64,32)$ & $6 \mathrm{Mb} / \mathrm{s}$ \\
\hline & & & $\operatorname{RS}(160,128)$ & $9.6 \mathrm{Mb} / \mathrm{s}$ \\
\hline \multirow[t]{5}{*}{ OOK } & 8B10B & $30 \mathrm{MHz}$ & $\operatorname{RS}(64,32)$ & $12 \mathrm{Mb} / \mathrm{s}$ \\
\hline & & $60 \mathrm{MHz}$ & $\mathrm{RS}(64,32)$ & $24 \mathrm{Mb} / \mathrm{s}$ \\
\hline & & $120 \mathrm{MHz}$ & $\operatorname{RS}(160,128)$ & $38.4 \mathrm{Mb} / \mathrm{s}$ \\
\hline & & & $\operatorname{RS}(160,128)$ & $76.8 \mathrm{Mb} / \mathrm{s}$ \\
\hline & & & None & $96 \mathrm{Mb} / \mathrm{s}$ \\
\hline
\end{tabular}

\section{Channel model}

For proper design and implementation, the characteristics of the channel must be understood. Characterization is performed by the channel impulse response, which is then used to analyze and combat the effects of channel distortions. In this scenario, the channel is de- fined between the Main LED unit (MLU) and Laptop, so it is an indoor channel. Two types of configurations may be considered according to the field of view (FOV) of the transmitter and receiver and secondly, the existence of a line of sight (LOS) path between the transmitter and the receiver. 
The link is considered to have LOS between the MLU and laptop. It does not suffer the effects of multipath fading, but there may be scattering effects due to reflections from objects.

These parameters are not simulated in this work. Since the distance between transmitter and receiver is small, the channel response $\mathrm{h}(\mathrm{t} ; \mathrm{S} ; \mathrm{R})$, where $\mathrm{S}$ and $\mathrm{R}$ are the positions of the transmitter and receiver respectively (Miramirkhani \& Uysal, 2020). This is approximately equal to that in indoor applications so defined as:

$h(t ; S ; R)=\sum_{k=0}^{\infty} h^{(k)}(t ; S ; R)$

where, $\mathrm{k}$ denotes the reflection order.

For $k=0, h(0)(t ; S ; R)$,

the expression is

$h^{(0)}(t ; S ; R) \approx \frac{n+1}{2 \pi} \cos ^{N}(\phi) d \Omega \pi(\theta / F O V) \delta\left(t-\left(\frac{d}{c}\right)\right)$ where, $N$ is the mode number that specifies the source of the direction. $(\phi, \theta)$ define the source radiation lobe. Field of View (FOV) is the solid angle through which the detector is sensitive to detect light.

This may be used to reduce unwanted reflections. $d \Omega$ is the receiver solid angle, $\mathrm{d} \approx \cos (\theta)\left({ }^{A_{\mathrm{r}}} / d^{2}\right)$ where $A_{\mathrm{r}}$ is the detector area and $d$ the distance between the transmitter and receiver. The symbol $\delta$ represents the delay of the beam. To calculate the kth reflection $h^{(k)}(t ; S ; R)=\int_{S} h^{(0)}\left(t ; S,\left\{r, \hat{n}, \pi / 2, d r^{2}\right\}\right) \otimes h^{k-1}(t ;\{r, \hat{n}, 1\}, R)$ The values of Table 4 , provided by the supplier (OLEDDCOM, 2018) can be used to determine the effect of the reflections on reception. The reflections do not create any problems for detection because the direct beam is much stronger than the reflected beams by physical observation.

TABLE 4

Transmitter Receiver Referential

\begin{tabular}{llll}
\hline Transmitter & & Receiver & $1 \mathrm{~cm}^{2}$ \\
& $\varnothing=-90^{\circ} \quad \theta=0^{\circ}$ & Detector Area $\left(A_{\mathrm{r}}\right)$ & \\
LED Power & 40 Watts & FOV & $\pm 30^{\circ}$ \\
S( x,y,x) & $2,5,2,5,3$ & R $(\mathrm{x}, \mathrm{y}, \mathrm{x})$ & $(1.5,1.5,0)$ \\
Reflectivity & 0.8 & Number of reflections & 3 \\
mode number $\mathrm{n}$ & 1 & $\mathrm{D}$ & $1 \mathrm{~m}$ \\
\hline
\end{tabular}

\section{Experimental}

The design methodology of the LiFi testbed is composed of four parts namely; prediction of the behavior and operating performance (data transmission rate) of the LEDs using mathematical model, (2) determination of the parameters of the photo transmitter, (3) determination of the parameters of the photoreceiver, and (4) Design principles and architecture. (Khanolkar, Menon, Band \& Rane,2013), proposed the mathematical model to predict the operating behavior of the LEDs, the data rate is influenced by the Size, the number, and the ON-OFF switching or rate of flickering of LEDs. Maximum data rates are achievable with smaller LEDs in larger quantities at a higher switching rate. Hence, the data rate (R) is directly proportional to the number of LEDs (Nled), the ON-OFF switching (Oled) of the LEDs, and inversely proportional to the LED size (Sled). LEDs are of different sizes in the order $1 \mathrm{~nm}, 1 \mu \mathrm{m}, 1 \mathrm{~mm}, 1.8 \mathrm{~mm}, 3 \mathrm{~mm}$, and 5 $\mathrm{mm}$ with maximum data rates achievable with the $1 \mu \mathrm{m}$ and $1 \mathrm{~nm}$ LEDs. The data transmission rate $(\mathrm{R})$, and the influencing parameters namely; (Oled), (Sled) and (Nled) are expressed by the relation; 


$$
R \propto N_{\text {led }} / S_{\text {led }}
$$

The data transmission by the photo transceiver is based on the assumption of direct line of sight (LOS) channels and simplex channel conditions and is not affected by other light sources as the receiver is only interested in the subtle changes of the transmitter (Khanolkar et al.,2013). Signal transmission can be achieved without direct LOS Communication since light reflected from the incident plane can also let us achieve reasonable speed. IEEE 802.15.7 networking employs random access frame acknowledgment, and data verification to increase the probability of a successful data transmission (I. C. Society, 2011).

\section{Testbed design principles and architecture}

In this section, we present our system design that integrates an IEEE 802.3 standard for wired Ethernet and a LiFi access point (LAP) that conforms to the IEEE 802.15.7 r1 standard for VLC. The designing methodology of the testbed architecture was constituted by three main building blocks namely; the transmitter also known as the main LED unit (MLU), a bi-directional LiFiNET LED TxRxLN-1MIP-PN panel embedded with a little microchip and provisioned with a wired Ethernet port to serve as the interface for the data source. The main functionality of the MLU is to act as the light-emitting source and an Ethernet to VLC converter. The numerical aperture or otherwise field of view FoV of the MLU is $\pm 30^{\circ}$ and uses RJ45 connectors. The LED panel has a dimension of $600 \mathrm{~mm} * 600 \mathrm{~mm} * 130 \mathrm{~mm}$ and a power output of 40 watts. It is powered by a $60 \mathrm{~W}$ multiple-stage Output Current LED Power Supply. The second block is an LED transceiver which is a bi-directional LiFiNET modem/USB dongle for USB to VLC signal conversion. The main functionality of the dongle is to receive the data stream from the MLU to the suitably adapted connected device, a LiFi enabled windows OS Lenovo L440 laptop, our preference for the study. The dongle front-end is equipped with dual Photosensors for transmitting and receive. These are protected by a transparent half-moon shaped fiberglass shield.

In this study, the LiFiNET LED panel was used to create the optical wireless signal access points (OWAPs) referred to as "attocells" for the testbed. The basic block diagram of the LiFi network is shown in Fig. 1.

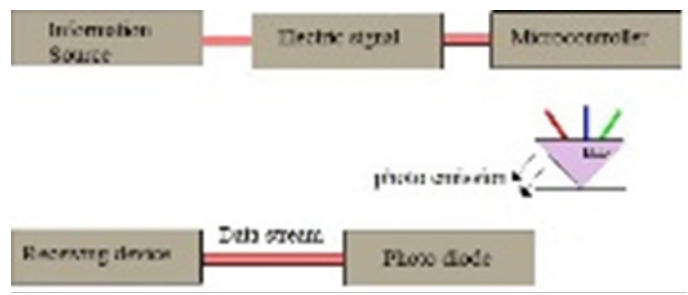

Fig.1: Basic LiFi Block diagram (Source: Authors own visualization).

\section{Setup of testbed}

The MLU was mounted to the ceiling of the testbed space at $0^{\circ}$ to the flooring. A TP-Link router served as a DHCP server to generate IP addresses for both an FTP server and the MLU. A one-meter length rod was used to calculate the distance in varying the length of the receiver from the MLU.

Finally, an on-device protractor was used to calculate the varying angular degrees we tested.

There were two scenarios of using a remote $\mathrm{ftp}$ server on the internet and a local ftp server setup on a windows 10 desktop. FileZilla was the ftp server application of choice. This server and the transmitter were both connected to the router to be on the same local network.

The measurement was taken at varying angular and lateral distances from the MLU. A constant alternating current (AC) of $0.65 \mathrm{~A}$ 
and an input voltage of $230 \mathrm{v}$ was applied to the base of the MLU to trigger the emission of a constant stream of photons observed as visible light. The received optical signal was then demodulated by the bi-directional LiFiNET modem/USB dongle attached to the laptop, then converted into a data stream to establish a point to point communication.

The Lenovo laptop installed with a windows $10-64$ bits operating system was powered and the necessary drivers that will enable the LiFiNET modem to detect the optical signals from the LED panel installed. The drivers used for the testbed are drivers for super-speed USB-LAN ICs. These are USB 2.0 to Fast Ethernet drivers for windows and IOS computers. For our application, we downloaded and used the AX88772C driver for windows 10 64-bit workstations. The two functional light fidelity real-time testbeds are shown in Figs. 2 and 3.
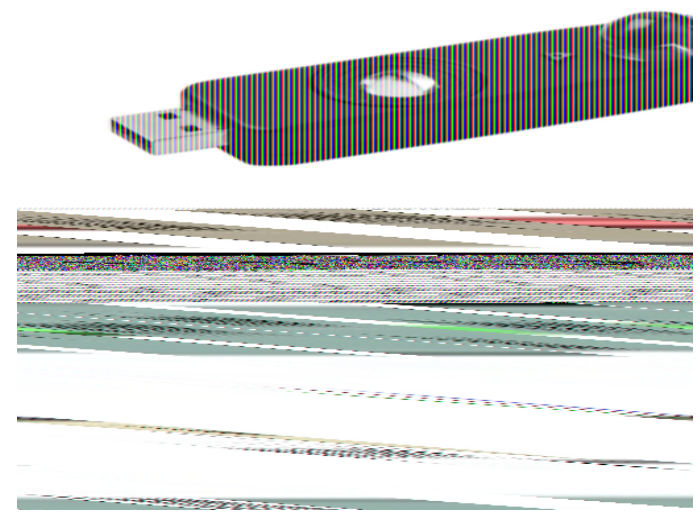

Fig. 2: Schematic LiFi testbed architecture with internet (Source: Oledcomm modified by the authors).

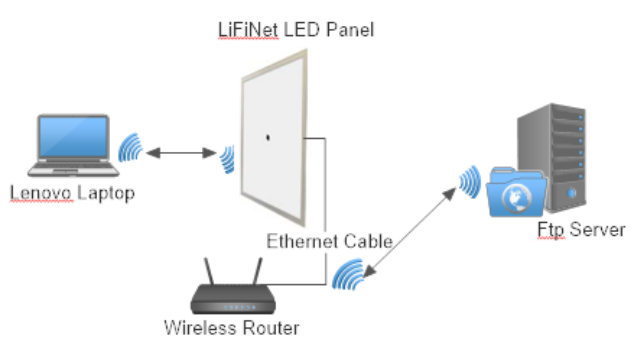

Fig. 3: Schematic LiFi testbed architecture without the internet (Source: authors own visualization).

\section{Testbed component details}

The quality of any study is enhanced by a good understanding of the research design.

\section{Hardware}

The LiFiNET Modem/ USB dongle

LiFiNET modem is a 2.0 bi-directional USB terminal connector. Based on VLC/LiFi communication standard protocols IEEE 802.15.7. Fig. 4 , is a photograph of the LiFiNET Modem.

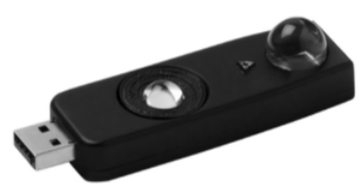

Fig. 4. LiFiNET modem (Source: Photo taken by the authors).

\section{LiFiNET LED Panel}

The LED Panel is compliant with visible light international communication standard IEEE 802.15.7 r1 and integrated with a router. It adapts to LED lighting (up to $100 \mathrm{~W}$ ) and allows secure high-speed Internet access (up to 2 Mbps) via a USB 2.0 terminal of the LiFiNET dongle to a Windows PC, MacBook, Linux, or Smartphone. It can be pictured as an Ethernet 
to a visible light communications converter. Fig. 5, shows the LED panel. Table 3, shows the main technical characteristics of the LED panel.

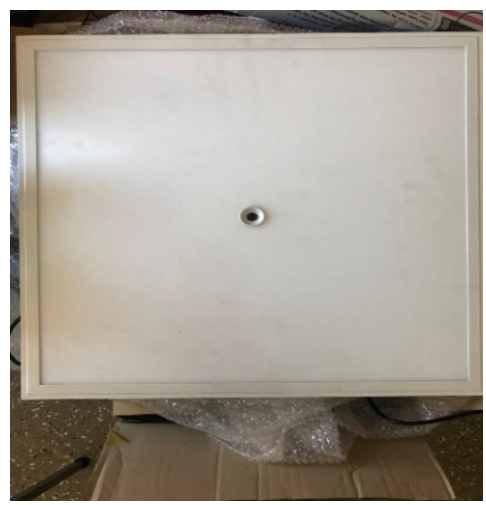

Fig. 5: LiFiNET LED Panel (Source: Photo taken by the authors).

TABLE 5

LiFiNET LED panel Technical Specification from supplier

\begin{tabular}{ll}
\hline SPECIFICATION & VALUE \\
\hline Data rates & 1 Mbps downlink + 1 \\
& Mbps uplink \\
Number of users & t user connected at a \\
& time
\end{tabular}

Communication range

Photosensor performances

Panel Standards

Panel Connectors

Dongle Standards

Dongle connectors

OS supported

Temperature surge

Voltage Surge

Temperature range

Humidity

Electromagnetic Field

Electrical Safety

Photo-biological Safety

Power

Length $\mathrm{x}$ Width

Height (maximal)

Color Temperature

Lumen of sight

Numerical aperture

$+/-30^{\circ}$

IEEE 802.15.7 (LiFi) and IEEE 802.3 (Ethernet)

RJ45

IEEE 802.15.7 (LiFi)

and USB 2.0

USB type A

Linux, Windows, Apple

Protected

Protected

$0^{\circ} \mathrm{C}$ to $70^{\circ} \mathrm{C}$

$10 \%$ to $80 \%$

EN55015; EN61547;

EN55022; EN55024

EN60598; EN61347;

EN60950

EN62471

$40 \mathrm{~W}$

$600 \mathrm{~mm} \times 600 \mathrm{~mm}$

$130 \mathrm{~mm}$

White: $4000-4500^{\circ} \mathrm{K}$

$3750 \mathrm{~lm}$
(Source: Oledcomm modified by authors)

\section{Results and discussion \\ The output of the tests are as shown in Table 6:}

Up to $1.8 \mathrm{~m}$ in the line

TABLE 6

Results of Tests Using Internet Connectivity

\begin{tabular}{|c|c|c|c|c|c|c|c|c|c|c|c|c|}
\hline \multirow[b]{3}{*}{$\begin{array}{l}\text { Download speed / } \\
\text { Mbps }\end{array}$} & \multicolumn{12}{|c|}{ Distance in Meters } \\
\hline & \multicolumn{6}{|c|}{1} & \multicolumn{6}{|c|}{2} \\
\hline & 0.86 & 0.72 & 0.88 & 0.83 & 0.86 & 0.78 & 0.83 & 0.91 & 0.89 & 0.96 & 0.93 & 0.77 \\
\hline $\begin{array}{l}\text { Upload speed / } \\
\text { Mbps }\end{array}$ & 0.07 & 0.59 & 0.07 & 0.62 & 0.51 & 0.3 & 0.07 & 0.21 & 0.45 & 0.05 & 0.46 & 0.20 \\
\hline Latency /ms & 42 & 36 & 38 & 32 & 30 & 32 & 41 & 37 & 29 & 33 & 37 & 41 \\
\hline Angles/ Degrees & $0^{\circ}$ & $30^{\circ}$ & $45^{\circ}$ & $90^{\circ}$ & $135^{\circ}$ & $180^{\circ}$ & $0^{\circ}$ & $30^{\circ}$ & $45^{\circ}$ & $90^{\circ}$ & $135^{\circ}$ & $180^{\circ}$ \\
\hline $\begin{array}{l}\text { LiFi Signal Re- } \\
\text { ception / Wm } \mathbf{m}^{-2}\end{array}$ & \multicolumn{6}{|c|}{3.183} & \multicolumn{6}{|c|}{0.253} \\
\hline
\end{tabular}


The angles depicted in Table 6 above are referencing the angle between the mount point of the illuminating optical transmitter and the receiving device.

The distances used were one and two meters respectively. Each distance was tested to determine download speed, upload speed, and the latency in transmission. The LiFi transceivers were positioned at various degrees apart within each of the respective distances mentioned. The angles for testing were specifically $0,30,45,90,135$, and 180 degrees for both one and two meters radius of testing.
The signal strength of the transceivers was also checked at the two respective distances. The output is shown in Table 6 .

\section{Download speeds}

It was observed download speeds of $0.86,0.72$, $0.88,0.83,0.86$, and 0.78 were recorded for the angles $0,30,45,90,135$ and 180 degrees respectively, evidently low was the download speed at 180 degrees within a one-meter radius. This averages a speed of $0.8217 \mathrm{Mbps}$ for download speed with a one-meter radius. Fig 6 . shows a visual representation of the output.

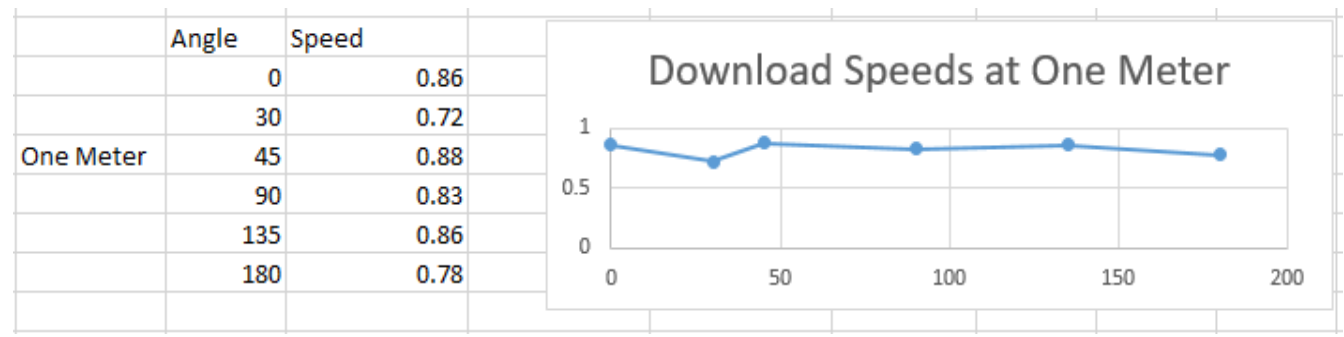

Fig. 6: Download Speeds at One Meter (Source: Researcher Lab Results).

It was observed download speeds of 0.83 , $0.91,0.89,0.96,0.93$, and 0.77 was recorded for the angles $0,30,45,90$, and 180 degrees respectively, evidently low was the download speed at 180 degrees within a two meters radius. This averages a speed of $0.8817 \mathrm{Mbps}$ for download speed with two meters radius. Fig 7. shows a visual representation of the output.

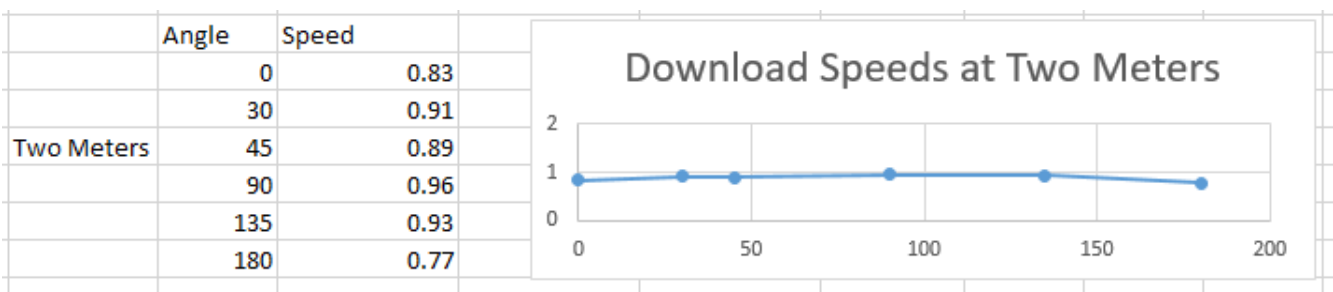

Fig. 7: Download Speeds at Two Meters (Source: Researcher Lab Results) 


\section{Upload speeds}

The upload speeds of $0.07,0.59,0.07,0.62$, 0.51 , and 0.30 was recorded for the angles 0 , $30,45,90,135$, and 180 degrees respectively within a meter radius. Evidently low was the upload speed at 180 degrees within a meter radius, $0.30 \mathrm{Mb}$ of data was uploaded per sec. The average upload speed is, therefore, 0.36 Mbps for a one-meter radius. Fig. 8. shows a visual representation of the output.

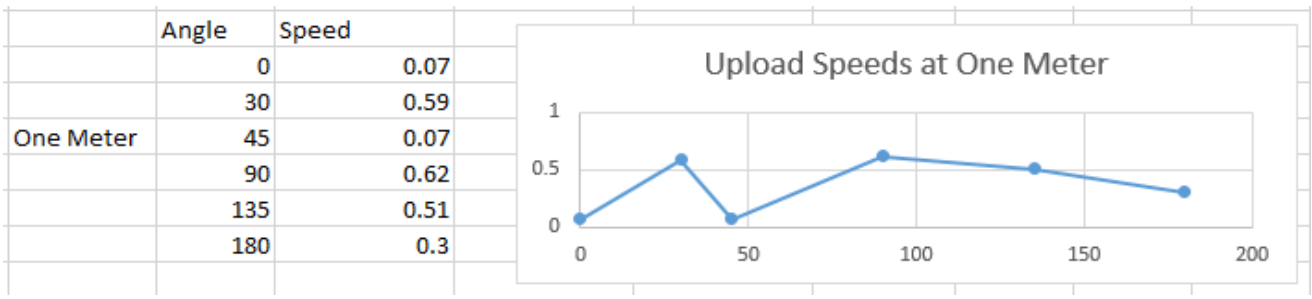

Fig. 8: Upload Speeds at One Meter (Source: Researcher Lab Results).

The upload speeds of $0.07,0.21,0.45,0.05, \quad$ low data of $0.2 \mathrm{Mb}$ was uploaded per sec. The 0.46 , and 0.20 were recorded for the angles 0 , average upload speed is, therefore, $0.24 \mathrm{Mbps}$ 30, 45, 90, 135, and 180 degrees, at 180 de- for two meters radius. Fig. 9. shows a visual grees respectively within two meters radius, representation of the output.

\begin{tabular}{|c|c|c|c|c|c|c|}
\hline & Angle & Speed & \multirow{2}{*}{\multicolumn{4}{|c|}{ Upload Speeds at Two Meters }} \\
\hline & 0 & 0.07 & & & & \\
\hline & 30 & 0.21 & & & & \\
\hline \multirow[t]{4}{*}{ Two Meters } & 45 & 0.45 & 0.4 & & & \\
\hline & 90 & 0.05 & 0.2 & & & \\
\hline & 135 & 0.46 & 0 & & & \\
\hline & 180 & 0.2 & 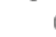 & 100 & 150 & 200 \\
\hline
\end{tabular}

Fig. 9: Download Speeds at Two Meter (Source: Researcher Lab Results).

\section{Latency experienced}

degrees respectively. This averages to $35 \mathrm{~ms}$ The latency of $42,36,38,32,30$, and 32 were latency within the one-meter radius. Fig. 10. recorded for angles $0,30,45,90,135$, and 180 shows a visual representation of the output.

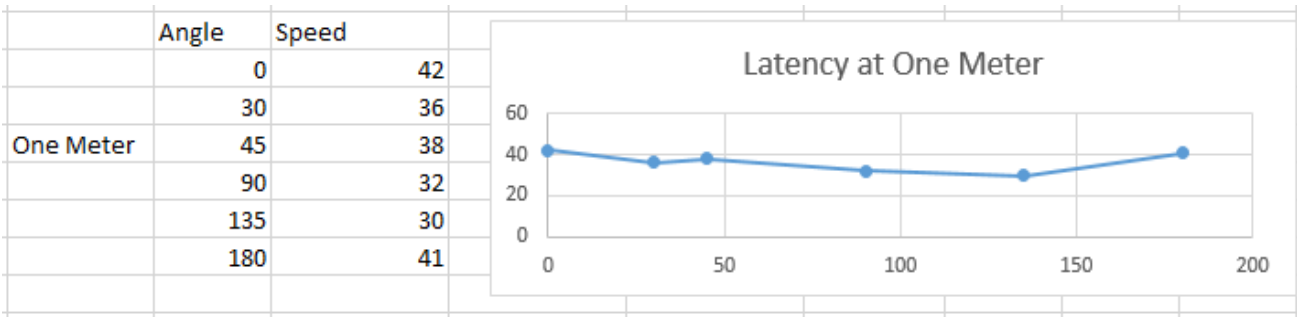

Fig. 10: Latency Speeds at One Meter (Source: Researcher Lab Results) 
The latency of 41, 37, 29, 33, 37, and 41 was recorded for angles $0,30,45,90,135$, and 180 degrees respectively. This averages to 35.33 ms within the two meters radius. Fig. 11. shows a visual representation of the output.

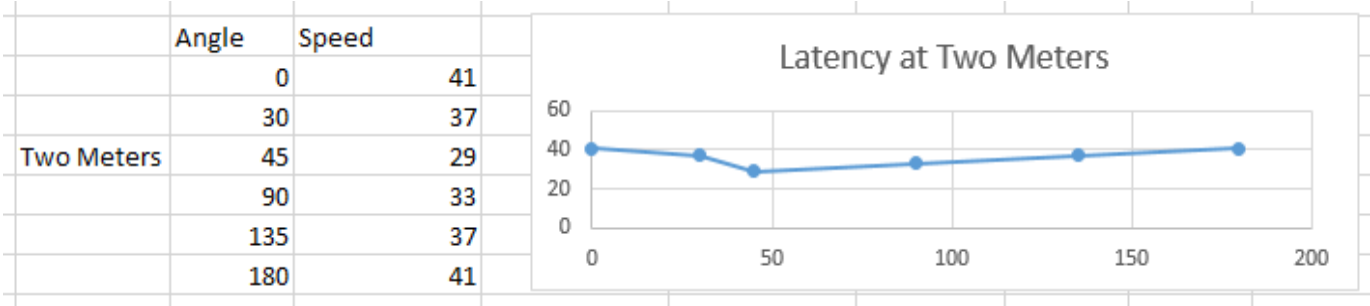

Fig. 11: Latency Speeds at Two Meters (Source: Researcher Lab Results).

\section{LiFi signal reception}

The signal strength of the LiFi Signal Reception averaged $3.183 \mathrm{Wm}^{-2}$ within a one-meter radius and $0.253 \mathrm{Wm}^{-2}$ within a two-meter radius.

Qualitative analysis of results

The overall results show that the latencies recorded were averagely very similar in speeds regardless of the distance or angle at which the data was transmitted. There was predominantly no established consistency in the pattern of latency experienced. Fig. 12. shows a visual representation of the output.

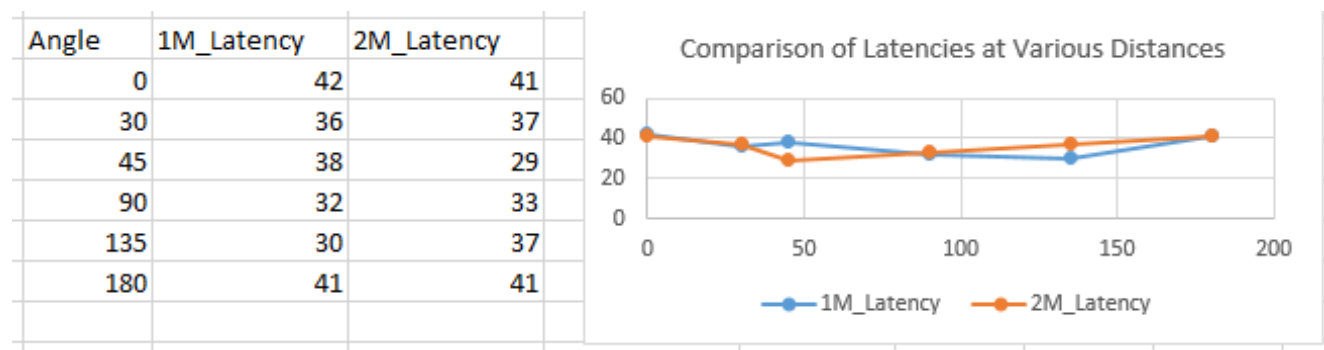

Fig. 12: Latency Speeds Comparison at Respective Angles, One \& Two Meters (Source: Researcher Lab Re-

sults).

There was, however, a distinct difference in average download and upload speeds to the angle of the transceivers' communication. The download speed was averagely double that of the upload speed despite both transmissions occurring over the same link. The significantly prominent observation was the seemingly same or very close speeds recorded at angles 0 and 180 degrees. Comparatively, the upload speeds are also relatively very low at angles 0 and 180 degrees. 


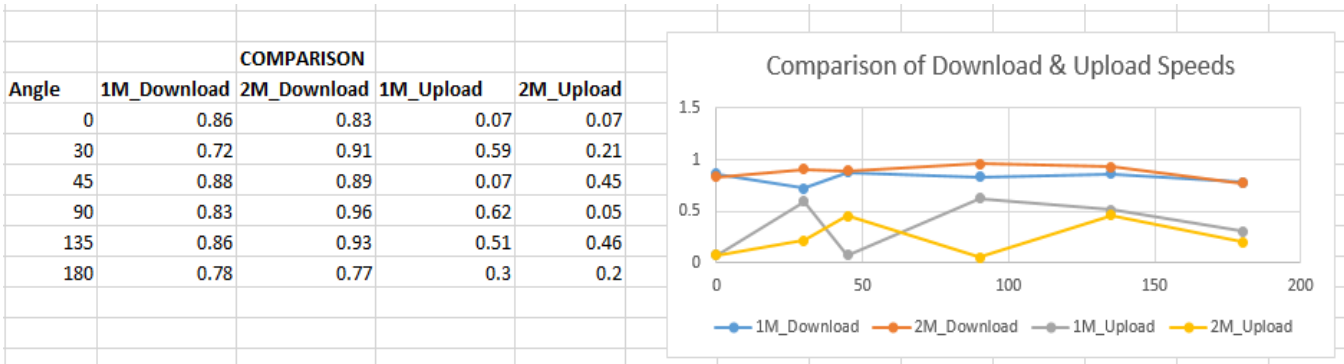

Fig. 13: Upload and Download Speeds Comparison at Respective Angles, One \& Two Meters (Source: Researcher Lab Results).

Results of additional tests carried out in a different setting are provided below, in this setting, a local FTP server is used hence connectivity at varying angles and distances are used to upload and download a file of the same size to ascertain the influence of distance and angular positioning on LiFi transmission speeds. The main objective of this optional test setting is to eliminate the possibility of unknown contention when transmitting via the internet.

Table 7 shows the recorded speeds at varying distances and angular positioning in the local setting.

TABLE 7

Results of Tests Using Local FTP Server

\begin{tabular}{llllllllllll}
$\begin{array}{l}\text { Data Transmission } \\
\text { Speed / Mbps }\end{array}$ & \multicolumn{10}{c}{ Distance in Meters } \\
\hline $\begin{array}{l}\text { Download speed } \\
\text { Mbps }\end{array}$ & 90 & 91 & 90 & 90 & 90 & 115 & Fail & 92 & 90 \\
$\begin{array}{l}\text { Upload speed /Mbps } \\
\text { Angles/ Degrees }\end{array}$ & 97 & 93 & 96 & 102 & 96 & 94 & Fail & 92 & 92 \\
& $\mathbf{3 0}^{\circ}$ & $\mathbf{6 0}^{\circ}$ & $\mathbf{9 0}^{\circ}$ & $\mathbf{3 0}^{\circ}$ & $\mathbf{6 0}^{\circ}$ & $\mathbf{9 0}^{\circ}$ & $\mathbf{3 0}^{\circ}$ & $\mathbf{6 0}^{\circ}$ & $\mathbf{9 0}^{\circ}$ \\
\hline
\end{tabular}

90, 91, 90, and 97, 93, 96 were recorded as download and upload speeds respectively for the angles 30,60 , and 90 within a meter radius. Fig. 14. shows a visual representation of the output. The general download speeds are averagely lower than the upload speeds in the one-meter radius from all angles.
Download and Upload Speeds at One Meter

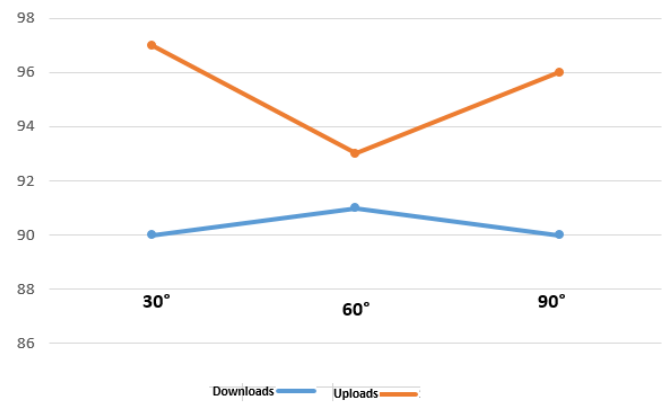

Fig. 14: Download and Upload speed at One Meter via Local FTP Server. 
90, 90, 115 and 102, 96, 94 were recorded as download and upload speeds respectively for the angles 30, 60, and 90 within a two meters radius. Fig. 15. shows a visual representation of the output. The general download speeds increase as the angle increases up to 90 degrees whereas the upload speeds decrease as the angle increase.

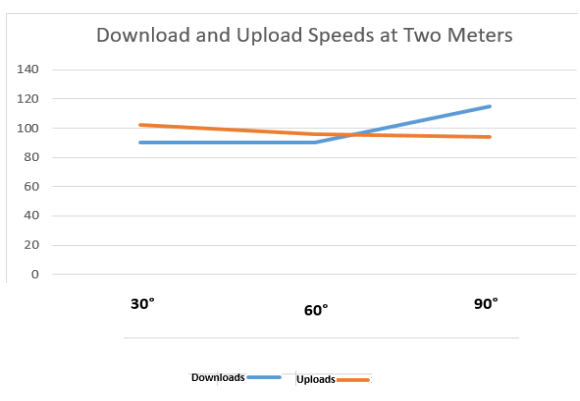

Fig. 15: Download and Upload speed at Two Meters via Local FTP Server.

92, 90, and 92, 92 was recorded as download and upload speeds respectively for the angles 60 and 90 within a three meters radius. Fig. 16. shows a visual representation of the output. There was no successful transmission at 30 degrees for either uploads or downloads. At three meters, it is observed that the general download speeds are at par for both downloads and uploads as the angle increases up to 90 degrees.

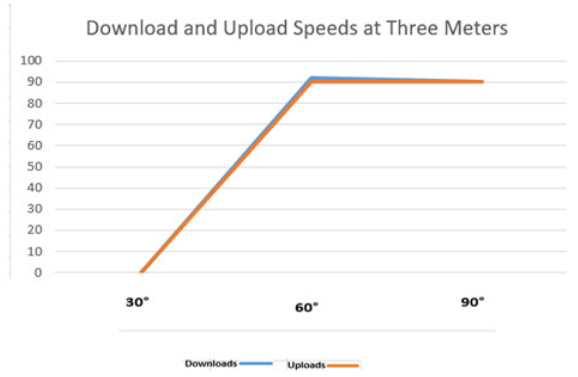

Fig. 16: Download and Upload speed at Three Meters via Local FTP Server.
The findings from the above analysis suggest that LiFi transmissions are not significantly influenced by distance and angular positioning for downloads and uploads. The only notable variation from this induction is however results for angular positioning for uploads, particularly in situations where the angles are specifically 0 or 180 degrees. This was only experienced in the setup with internet connectivity. It is essential to indicate in this context that the possible interference or obstruction of the environment was not considered in this research. There was no attempt in the experiment to cause any form of interference by positioning other transceivers at various angles to interfere with the measured transceivers. The measurements are as indicated in the methodology section of this paper based on a tool from a remote location on the internet and also locally in the second setting. The implication is that the results obtained are subject to possible content beyond the gateway for internet connectivity. Locally, however, the experiment did not show any significant variation and consistent pattern in speeds for uploads, downloads, and the various distances.

\section{Conclusion}

In this paper, the flexibility of LiFi technology in terms of its efficiency and effectiveness over a distance of transmission and angular positioning of transceivers and illuminating devices using a single optical wireless access point is demonstrated. The testbed is proposed for an indoor scenario for which the results have been validated. The essential finding suggests that neither distance nor angular positioning impact speeds significantly as long as devices work within supported device ranges. The findings suggest that for optimal upload speeds in LiFi transmission, the angles of 0 or 180 degrees are not ideal for single optical access points due to the low transmission speeds observed. Other angles ranging from 30 to 
135 degrees are relatively better for optimal upload speeds. The findings, however, do not provide any prominently striking indication of best angles and distance for download speeds. The findings essentially suggest that the transmission of LiFi is not influenced by distance and angular positioning for both downloads and uploads over different distances. It is suggested that future research is carried out on LiFi with considerations of multiple access points to enhance the data density significantly through spatial reuse of bandwidth.

The benefits of LiFi are well established, some well-known benefits are the use of an LED bulb, thus not requiring any other special lighting and also used as a source of light. This implies it is efficient in terms of costs, energy-saving, and essentially available anywhere there is a light source. It is relatively secure because of its accessibility only by users within the reach of the light illumination.

LiFi is a technological revolution capable of influencing a great number of industries. LiFi can create new cyber-secure wireless networks, drive applications in Industry 4.0, unlock the IoT, and allow Light as a service (LaaS) in the lighting industry. It can contribute to the fifth-generation cellular networks $(5 \mathrm{G})$ and beyond thereby acting as an enabler for new smart transport systems to improve future road safety with the emergence of driverless cars and offer new solutions to close the digital divide.

\section{References}

Bokefode, J. D., Ubale, S. A. \& Gaikwad, R. M. (2018) Retrieving Real Time Data through IOT Devices and Storing Securely on Cloud Using LiFi, 2018 3rd International Conference for Convergence in Technology, I2CT 20188529393.

CISCO ANNUAL INTERNET REPORT (2018) Annual White Paper, Retrieved from https:// www.cisco.com/c/en/us/solutions/collateral/ executive-perspectives/annua internet-report/ white-paper-c11-741490.html.[Accessed May $18,2020]$.

HaAs, H.(2013) "High-speed wireless networking using visible light," Society of Photographic Instrumentation Engineers (SPIE), Volume 10.1117/2.1201304.004773, pp. 1 - 3.

IEEE Computer Society (2017) "Part 15.7: ShortRange Wireless Optical Communication Using Visible Light," in IEEE Standard for Local and metropolitan area networks, New ork, IEEE Computer Society, 2011, pp. 1 - 286.

Islam, R. \& Mondal, M. R. H. (2019) Hybrid DCOOFDM, ACO-OFDM and PAM-DMT for dimmable LiFi, Optik 180, 939 - 952.

Kamsula, P. (2015) "Desing and implementation of a bi-directional visible light communication testbed". The University of Oulu, Department of Electrical and Information Engineering. Master's Thesis.

Khanolkar, N., Menon, K. B \& Rane C. (2013) "Visible Light Data Transmission," AY 2012 2013.

MAHENDRAN, R. (2017) Integrated LiFi (Light Fidelity) for smart communication through illumination, Proceedings of 2016 International Conference on Advanced Communication Control and Computing Technologies, ICACCCT 20167831599 , pp. $53-56$.

MARKETS AND MARKETS (2018) Market Report, Retrieved from https://www.marketsandmarkets.com /Market-Reports/visible-light-communication-market-946.html. [Accessed May 21, 2020.2020].

Miramirkhani, F. \& Uysal, M. (2020) Channel modelling for indoor visible light communications. Philos Trans A Math Phys Eng Sci. 378 (2169):20190187. doi:10.1098/ rsta.2019.0187.

Monisha, M. \& SudheEndra, G. (2018) LiFi-Light Fidelity Technology, International Conference 
on Current Trends in Computer, Electrical, Electronics and Communication, CTCEEC 2017 8455097, pp. 818 - 821.

OLEDCOMM, L. (2017) Technical Datasheet LiFiNET LED panel Ref.: TxRx-LN-1M-IPPN. Retrieved from https://www.oledcomm. net/category/products/lifinet. [Accessed July, 10 2019].

Pikasis, E. \& Popoola, W.o. (2018) Understanding LiFi Effect on LED Light Quality, 31st Annual Conference of the IEEE Photonics Society, IPC 20188527234.

Pikasis, E. \& Popoola, W. O. (2018) Understanding LiFi Effect on LED Light Quality, IEEE
Photonics Conference (IPC), 1 - 2. 10.1109/ IPCon.2018.8527234.

Punthawanunt, S., Aziz, M. S., Phatharacorn, P., Ali, J. \& Yupapin, P. (2018) LiFi cross-connection node model using whispering gallery mode of light in a microring resonator, Microsystem Technologies 24 (12), 4833 - 4838.

Singh, D., Sood, A., Thakur, G., Arora, N. \& Kumar, A. (2017) Design and implementation of wireless communication system for toll collection using LiFi, 4th IEEE International Conference on Signal Processing, Computing and Control, ISPCC 2017 2017-January, pp. 510 - 515].

Received 15 Aug 19; revised 22 Jul 20. 